\title{
Shedding light on LMA-dark solar neutrino solution by medium baseline reactor experiments: JUNO and RENO-50
}

\section{P. Bakhti and Y. Farzan}

School of physics, Institute for Research in Fundamental Sciences (IPM), P.O.Box 19395-5531, Tehran, Iran

E-mail: pouya_bakhti@ipm.ir, yasaman@theory.ipm.ac.ir

ABSTRACT: In the presence of Non-Standard neutral current Interactions (NSI) a new solution to solar neutrino anomaly with $\cos 2 \theta_{12}<0$ appears. We investigate how this solution can be tested by upcoming intermediate baseline reactor experiments, JUNO and RENO-50. We point out a degeneracy between the two solutions when both hierarchy and the $\theta_{12}$ octant are flipped. We then comment on how this degeneracy can be partially lifted by long baseline experiments sensitive to matter effects such as the NOvA experiment.

Keywords: Neutrino Physics, Solar and Atmospheric Neutrinos

ARXIV EPRINT: 1403.0744 


\section{Contents}

1 Introduction 1

2 Oscillation probability 2

$3 \quad$ JUNO and RENO-50 experiments 3

$4 \quad$ Numerical results $\quad 5$

5 Degeneracy and matter effects $\quad 6$

$\begin{array}{llr}6 & \text { Conclusions } & 9\end{array}$

\section{Introduction}

Within the Standard Model (SM) the neutral current interactions are flavor diagonal and universal for all three flavors. However, most beyond SM mechanisms dealing with flavor predict a correction to neutrino interaction terms which violate flavor universality and conservation. Examples of such models include $R$-parity violating supersymmetry, grand unification, AMEND model [1], extra $\mathrm{U}(1)^{\prime}$ gauge models, left-right symmetric models and various seesaw models (for a review see [2]). The non-standard neutral current interaction of neutrinos can be in general formulated by an effective dimension six operator as

$$
\mathcal{L}_{N S I}=-2 \sqrt{2} G_{F} \epsilon_{\alpha \beta}^{f P}\left(\bar{\nu}_{\alpha} \gamma^{\mu} L \nu_{\beta}\right)\left(\bar{f} \gamma_{\mu} P f\right)
$$

where $f$ is the matter field $(u, d$ or $e), P$ is the chirality projection matrix and $\epsilon_{\alpha \beta}^{f P}$ is a dimensionless matrix describing the deviation from the standard model. For neutrino oscillation, only the "vector" part of the interaction operator is relevant so it is convenient to define

$$
\epsilon_{\alpha \beta}^{f} \equiv \epsilon_{\alpha \beta}^{f L}+\epsilon_{\alpha \beta}^{f R} .
$$

Effects of Lagrangian (1.1) on neutrino oscillation have been extensively studied in the literature. In particular in [3-5], it is shown that in the presence of a deviation from universality (i.e., $\left|\epsilon_{e e}^{f}-\epsilon_{\mu \mu}^{f}\right|,\left|\epsilon_{e e}^{f}-\epsilon_{\tau \tau}^{f}\right| \neq 0$ with $f=u, d$ ), another solution with $\cos \left(2 \theta_{12}\right)<$ 0 for solar and KamLAND data exists. This solution is known as LMA-Dark solution. Recent studies show that this new solution survives combining all the available data on oscillation [6]. In fact in presence of Non-Standard Interactions (NSI), the fit to solar data is slightly better as in the presence of NSI, the upturn of the spectrum at low energy predicted by the standard LMA solution without NSI can be suppressed, leading to a better agreement with the data [3-5]. The NSI can also affect other observable quantities such as the invisible decay width of the $Z$ boson (at one-loop) or neutrino scattering off 
matter. All relevant bounds have extensively been studied $[2,7,8]$. The bound from the CHARM scattering experiment combined with the $\mathrm{NuTeV}$ results rule out a part of the parameter space relevant for the LMA-Dark solution (i.e., $0.9<\left|\epsilon_{e e}^{d}-\epsilon_{\mu \mu}^{d}\right|<0.8$ at 90 $\%$ C.L.) $[6,8]$. However, the LMA-Dark solution is not completely ruled out and needs further investigation.

Recently, two intermediate baseline neutrino experiments JUNO and RENO-50 have been proposed to be built in China and south Korea, respectively. Determining the neutrino mass hierarchy (i.e., normal vs inverted) and precision measurement of the solar mixing parameters $\theta_{12}$ and $\Delta m_{21}^{2}$ are the prime goals of these experiments [9-17] (see also [18-24]). Recently, we have shown that the data from these two experiments can also be employed to probe the superlight sterile neutrino scenario [25]. Refs. [26, 27] study the effects of charged current NSI at detector and source. The aim of the present paper is to show that the medium baseline experiments can help to probe the LMA-Dark solution for which $\theta_{12}>\pi / 4$. We find a degeneracy between solutions when signs of both $\cos 2 \theta_{12}$ and $\Delta m_{31}^{2}$ are simultaneously flipped and then discuss the possibility of lifting this degeneracy.

The rest of the paper is organized as follows. In section 2 , we review the oscillation probability and discuss how the medium baseline reactor experiments distinguish between the LMA and LMA-Dark solutions. In section 3, we describe the JUNO and RENO50 experiments and list the background. In section 4, we present our numerical results obtained via the GloBES software [28-30]. In section 5, we discuss the degeneracy in more detail and examine the possibility of lifting it. In section 6 , we summarize our results.

\section{Oscillation probability}

The energy of the reactor neutrinos are of order of $\mathrm{MeV}$ so in the leading order, the matter effects can be neglected in the propagation of these neutrinos in the earth (i.e., $\left.\Delta m_{21}^{2} / E_{\nu} \gg \sqrt{2} G_{F} N_{e}\right)$. As a result, the effect of neutral current NSI in eq. (1.1) on neutrino propagation can also be neglected. In fact, refs. [26, 27] focus on the charged current NSI that affect production and detection [i.e., $\left.\left(\bar{d} \gamma^{\mu} P u\right)\left(\bar{e} \gamma_{\mu} L \nu_{\mu(\tau)}\right)\right]$. Neutral current interaction of type (1.1) cannot affect the production and detection either. At first sight, it seems counterintuitive that reactor neutrinos help us to probe the impact of neutral current NSI. Notice however that we are proposing to determine $\cos 2 \theta_{12}$ rather than constraining the NSI parameters, $\epsilon_{\alpha \beta}^{f}$. Neglecting the matter effects, one can write

$$
\begin{aligned}
P\left(\bar{\nu}_{e} \rightarrow \bar{\nu}_{e}\right)= & \left.|| U_{e 1}\right|^{2}+\left|U_{e 2}\right|^{2} e^{i \Delta_{21}}+\left.\left|U_{e 3}\right|^{2} e^{i \Delta_{31}}\right|^{2}=\left|c_{12}^{2} c_{13}^{2}+s_{12}^{2} c_{13}^{2} e^{i \Delta_{21}}+s_{13}^{2} e^{i \Delta_{31}}\right|^{2} \\
= & c_{13}^{4}\left(1-\sin ^{2} 2 \theta_{12} \sin ^{2} \frac{\Delta_{21}}{2}\right)+s_{13}^{4} \\
& +2 s_{13}^{2} c_{13}^{2}\left[\cos \Delta_{31}\left(c_{12}^{2}+s_{12}^{2} \cos \Delta_{21}\right)+s_{12}^{2} \sin \Delta_{31} \sin \Delta_{21}\right]
\end{aligned}
$$

where $\Delta_{i j}=\Delta m_{i j}^{2} L /\left(2 E_{\nu}\right)$ in which $L$ is the baseline. For short baseline reactor experiments such as Daya Bay, RENO or (double-)CHOOZ, we can set $\Delta_{21} \simeq 0$ so the sensitivity to $\theta_{12}$ is lost altogether. At KamLAND, $\Delta_{21}$ is sizeable but the oscillatory modes given by $\Delta_{31}$ are averaged out so KamLAND is only sensitive to $\sin ^{2} 2 \theta_{12}$ which cannot distinguish 
between the two solutions with $\theta_{12}>\pi / 4$ and $\theta_{12}<\pi / 4$. To distinguish between the standard LMA and LMA-dark solutions the experiment should be sensitive to the last terms in eq. (2.1) given by $\cos \Delta_{31} \cos \Delta_{21}$ and $\sin \Delta_{31} \sin \Delta_{21}$. The JUNO and RENO-50 experiments are proposed to resolve these terms as the term given by $\sin \Delta_{31} \sin \Delta_{21}$ is the one sensitive to $\operatorname{sign}\left(\Delta_{31}\right)$ and hence the mass hierarchy scheme. In principle, by studying the energy spectrum of the events, we can resolve these terms and extract their amplitude and sign. Thus, we can discriminate between the standard LMA and non-standard LMA-Dark solutions. However, it is a non-trivial question to determine whether this can in principle be possible taking into account the realistic uncertainties. In the rest of the paper, we try to address this question. Before proceeding further notice that $P\left(\bar{\nu}_{e} \rightarrow \bar{\nu}_{e}\right)$ in eq. (2.1) is invariant under

$$
s_{12} \leftrightarrow c_{12}\left(\text { i.e., } \theta_{12} \rightarrow \frac{\pi}{2}-\theta_{12}\right) \quad \text { and } \quad \Delta_{31} \rightarrow-\Delta_{31}+\Delta_{21} .
$$

In other words, as far as we neglect matter effects, there is a degeneracy when we simultaneously flip hierarchy $(\mathrm{NH} \leftrightarrow \mathrm{IH})$ and flip between the LMA and LMA-Dark solutions. We will discuss more about this degeneracy in section 4 and in section 5, we will generalize this symmetry to include matter effects.

\section{JUNO and RENO-50 experiments}

The JUNO and RENO-50 experiments with baselines of $L \sim 50 \mathrm{~km}$ are scheduled to become ready for data taking in 2020 [31]. The detectors will use liquid scintillator technique with an energy resolution of

$$
\frac{\delta E_{\nu}}{E_{\nu}} \simeq 3 \% \times\left(\frac{E_{\nu}}{\mathrm{MeV}}\right)^{1 / 2} .
$$

Ref. [12] enumerates the following backgrounds as the dominant ones (i) accidental background; (ii) ${ }^{13} C(\alpha, n){ }^{16} O$ background and (iii) Geoneutrino background. For the spectrum of these sources of background and their normalization we use values and description respectively in [32] and in [12]. However, as shown in recent paper [33], the background caused by ${ }^{9} \mathrm{Li}$ from cosmic muon interaction will be dominant. We take 10000 and 5000 fake neutrino signals due to ${ }^{9} \mathrm{Li}$ at respectively JUNO and RENO-50 and assume a spectrum of shape given in [34] for them. The reason why the cosmic muon induced ${ }^{9} \mathrm{Li}$ background is substantially less for RENO-50 than that for JUNO is the deeper location of RENO-50 detector and therefore better shielding from cosmic muons. Notice that the normalization we take for ${ }^{9} \mathrm{Li}$ background is relatively conservative. Reconstructing the muon tracks and using a smart veto, the background can be reduced down to half the assumed value [35].

We divide the energy range between $1.8 \mathrm{MeV}$ to $8 \mathrm{MeV}$ to 350 bin of size $17.7 \mathrm{keV}$ in our analysis. We take the energy calibration error equal to $3 \%$. Let us now describe the features specific for each experiment one by one.

The JUNO experiment. JUNO will be located at a distance of $52 \mathrm{~km}$ far from Yangjiang and Taishan reactor complexes with a combined power of $36 \mathrm{GW}$ [11]. JUNO will also receive neutrino flux from the existing Daya Bay and planned Huizhou reactors respectively 


\begin{tabular}{|c|c|c|c|c|}
\hline reactor core & 1 & 2 & 3 & 4 \\
\hline Baseline $(\mathrm{km})$ & 52.17 & 52.36 & 52.58 & 52.80 \\
\hline Power $(\mathrm{GW})$ & 10.4 & 7.5 & 7.5 & 10.4 \\
\hline
\end{tabular}

Table 1. Baselines and powers of reactor cores taken for the JUNO experiment.

located $215 \mathrm{~km}$ and $265 \mathrm{~km}$ far from it. We take the flux normalization uncertainty to be $5 \%$. The scintillator detector will have a fiducial mass of 20 kton. A list of reactor distances and powers can be found in [11]. To simplify computation, in our numerical analysis we combine the reactor cores whose distance to detector are close to each other. Table 1 summarizes the powers and baselines that we take in our analysis.

The RENO-50 experiment. The RENO-50 setup is an upgrade of the current RENO experiment using the neutrino flux from the same reactors with a total power of $16.4 \mathrm{GW}$. The current detector will be used as near detector reducing the flux uncertainty down to $0.3 \%$ [36]. The far detector with a fiducial mass of $18 \mathrm{kton}$ will be located $47 \mathrm{~km}$ away.

The potential of reactor neutrino experiments with a baseline of $\sim 50 \mathrm{~km}$ for determining the neutrino mass ordering has been extensively studied in the literature [11, 1315, 18-24]. The main goal of JUNO and RENO-50 experiments is determining the sign of $\Delta m_{31}^{2}$. It is shown that in order to determine $\operatorname{sgn}\left(\Delta m_{31}^{2}\right)$, the difference between the distances of different reactor cores contributing to the flux of the detector should be less than $\mathcal{O}(500)$ meters $[11,14,17]$. Considering this restriction, the best location for JUNO is found to be at a $52 \mathrm{~km}$ distance from Yangjiang and Taishan reactor complexes [10, 11]. Like the case of determining the hierarchy, we expect the distribution of reactor sources to reduce the sensitivity to $\operatorname{sign}\left(\cos 2 \theta_{12}\right)$ because the distribution of the sources lead to average out of the effects of the oscillatory terms given by $\Delta_{31}$. Although the matter effects are subdominant, in the numerical analysis we take them into account.

From eq. (2.1), we observe that the terms sensitive to $\operatorname{sign}\left(\cos 2 \theta_{12}\right)$ are suppressed by $s_{13}^{2} \sim 2.5 \%$. Thus, at first glance it seems that an uncertainty of $3 \%$ or larger in the shape of the initial energy spectrum can wash out the sensitivity to $\operatorname{sign}\left(\cos 2 \theta_{12}\right)$ as well as the sensitivity to $\operatorname{sign}\left(\Delta m_{13}^{2}\right)$. In fact, the uncertainty in the shape of the initial energy spectrum at source is at the level of $O(3 \%)[37,38]$. However as we discuss below, the effects of this uncertainty can be safely neglected. Let us denote the uncertainty in the shape of the initial energy spectrum at energy bin " $i$ " by $\Delta \alpha_{i}$. We take into account the effect of this uncertainty by pull method, defining

$\chi^{2}=\left.\operatorname{Min}\right|_{\theta_{\text {pull }}, \alpha_{i}}\left[\sum_{i} \frac{\left[N_{i}\left(\theta_{0}, \bar{\theta}_{\text {pull }}\right)-N_{i}\left(\theta, \theta_{\text {pull }}\right)\left(1+\alpha_{i}\right)\right]^{2}}{N_{i}\left(\theta_{0}, \bar{\theta}_{\text {pull }}\right)}+\sum_{i} \frac{\alpha_{i}^{2}}{\left(\Delta \alpha_{i}\right)^{2}}+\frac{\left(\theta_{\text {pull }}-\bar{\theta}_{\text {pull }}\right)^{2}}{\left(\Delta \theta_{\text {pull }}\right)^{2}}\right]$,

where $\alpha_{i}$ is the pull parameter taking care of the uncertainty in the initial spectrum at bin $i$. $\theta_{\text {pull }}$ collectively denotes pull parameters other than $\alpha_{i}$ which have true values collectively denoted by $\bar{\theta}_{\text {pull }}$ and uncertainties collectively denoted by $\Delta \theta_{\text {pull }} . \theta$ and $\theta_{0}$ are respectively the fit parameter and its true value. $N_{i}$ is the number of events at bin $i$. To calculate the deviation, we minimize over each $\alpha_{i}$ as well as over all $\theta_{\text {pull }}$. It is straightforward to show 
that as long as

$$
N_{i}\left(\Delta \alpha_{i}\right)^{2} \ll 1,
$$

we can neglect the effects of $\Delta \alpha_{i}$ in evaluating $\chi^{2}$. Considering Fig (13) of ref. [39] and uncertainties found in $[37,38]$, we observe that even with spectrum divided into bins of size $17.7 \mathrm{keV}$, the condition in (3.2) is fulfilled so the present uncertainty in the shape of the spectrum will not be a major limitation for extracting $\operatorname{sign}\left(\cos \left(2 \theta_{12}\right)\right)$ and/or $\operatorname{sign}\left(\Delta m_{31}^{2}\right)$.

To carry out our analysis, we employ the GLoBES software [28-30]. We use the reactor neutrino energy spectrum and neutrino cross section that are respectively given in [40,41] and [42]. For neutrino mass and mixing parameters, we take the best fit values listed in [43]. We assume an uncertainty of $6 \%$ both in $\theta_{13}$ and in $\Delta m_{21}^{2}$. We use the pull-method to treat the uncertainties.

\section{$4 \quad$ Numerical results}

Figures 1, 2 show the potential of JUNO and RENO-50 experiments in determining both hierarchy and $\operatorname{sign}\left(\cos 2 \theta_{12}\right)$ after five years of data taking. We have assumed normal hierarchy and have taken the true value of $\theta_{12}$ to be equal to $\theta_{12}=33.57^{\circ}$ in figure 1 and equal to $\theta_{12}=56.43^{\circ}$ in figure 2. Contours show the $3 \sigma$ C.L. solutions. Notice that the determination of $\left|\Delta m_{31}^{2}\right|$ by either of these experiments will be far more precise than what is obtained by global analysis of the present data both in the absence of NSI [43] and in its presence [6]. They can also remarkably improve the precision on $\theta_{12}$. After five years of data taking, the precision of $\theta_{12}$ will reach a remarkable value of $\Delta \theta_{12}= \pm 0.4^{\circ}$ or better at $3 \sigma$ C.L. For ruling out the wrong hierarchy, we have checked our result against that in ref. [13] and it seems our results are in agreement.

From figure 1-a and figure 2-b, we observe that JUNO can determine these parameters more precisely than RENO-50 would. This is mainly due to the fact that the reactor power and therefore neutrino flux are higher at JUNO. As seen from figures 2 -a and -d, while at $3 \sigma$ RENO-50 finds solutions with wrong $\operatorname{sign}\left(\Delta m_{31}^{2}\right)$ or wrong $\operatorname{sign}\left(\cos 2 \theta_{12}\right)$, JUNO rules out these wrong solutions. We have found that when LMA-Dark is taken as the true solution, RENO-50, JUNO and their combined results rule out the wrong LMA solution with $\chi^{2}=5.5$ (i.e., $>90 \%$ C.L.), $\chi^{2}=12.9$ (i.e., $\sim 3 \sigma$ C.L.) and $\chi^{2}=19.94$ (i.e., $\sim 4 \sigma$ C.L.), respectively. Similarly for standard LMA solution with $\cos 2 \theta_{12}>0$, RENO-50, JUNO and their combined results rule out the wrong LMA-Dark solution with $\chi^{2}=4.95$ (i.e., $>90 \%$ C.L.), $\chi^{2}=11.4$ (i.e., slightly less than $3 \sigma$ C.L.) and $\chi^{2}=18.34$ (i.e., slightly less than $4 \sigma$ C.L.), respectively. Turning off the background, JUNO can also rule out the wrong LMA-Dark solution at more than $3 \sigma$ C.L. From figures 1 and 2, we also see that the precision by JUNO is overally better. Remember that we had assumed similar calibration uncertainty, energy resolution and background for these two experiments. By varying the calibration error by a factor of two we have found that the results from these two setup do not change much. However, as expected, similarly to the case of hierarchy determination $[15,16]$ the results are very sensitive to the energy resolution. For example, if we change the energy resolution from $3 \%\left(E_{\nu} / \mathrm{MeV}\right)^{1 / 2}$ to $3.5 \%\left(E_{\nu} / \mathrm{MeV}\right)^{1 / 2}$, the wrong solution becomes acceptable at $3 \sigma$ C.L. by combined five years data of JUNO and RENO-50. 

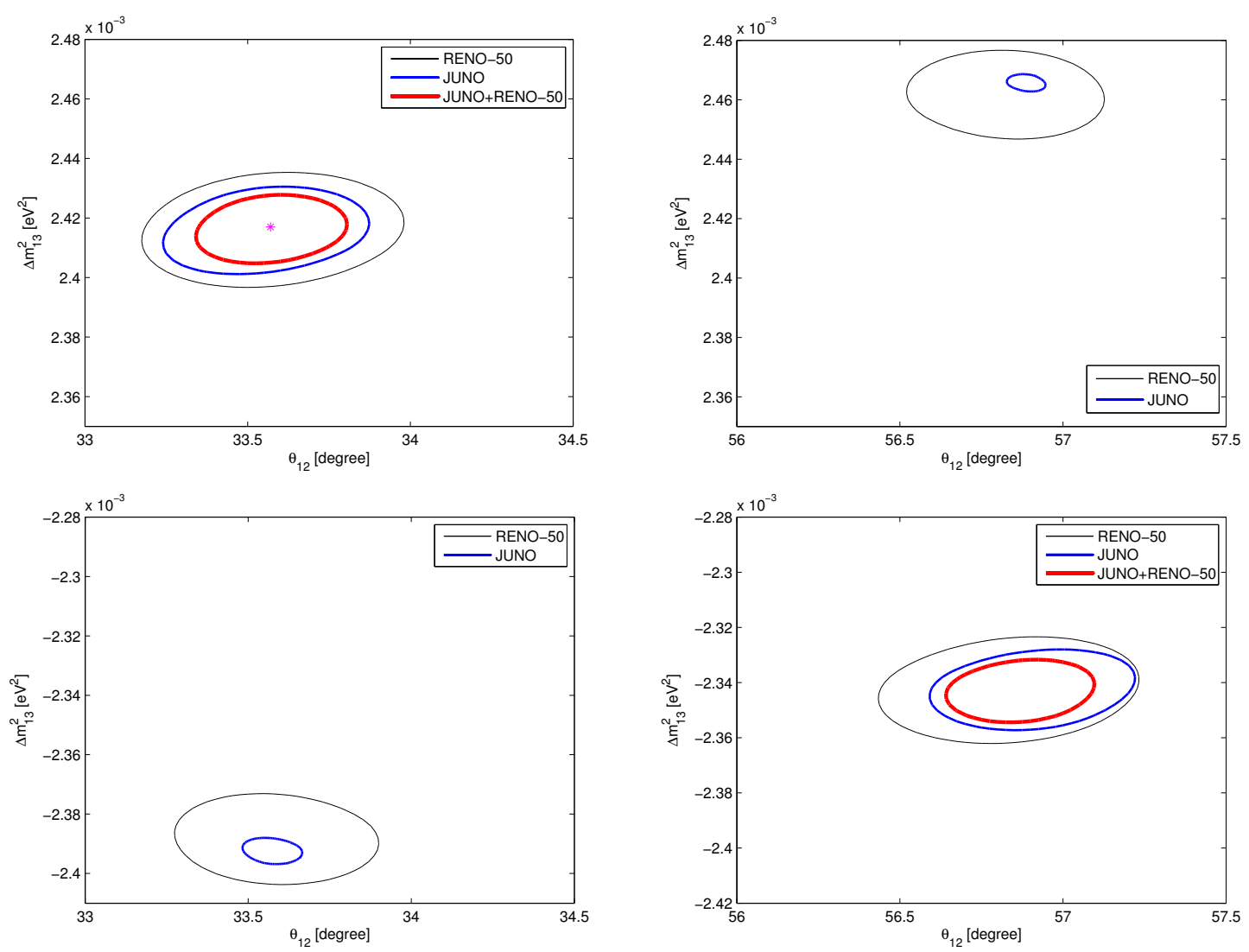

Figure 1. Allowed region at $3 \sigma$ C.L. after 5 years of data taking by RENO-50 and JUNO. The true values of the neutrino parameters, marked with a star in figure (a), are taken to be $\Delta m_{31}^{2}=2.417 \times 10^{-3} \mathrm{eV}^{2}, \theta_{12}=33.57^{\circ}, \Delta m_{21}^{2}=(7.45 \pm 0.45) \times 10^{-5} \mathrm{eV}^{2}$ and $\theta_{13}=(8.75 \pm 0.5)^{\circ}$. The upper (lower) panels show the allowed region for normal (inverted) hierarchy and left (right) panels show LMA (LMA-Dark) solution for $\theta_{12}$.

As seen from the figures 1 , the reactor experiments cannot distinguish between the solution with $\cos 2 \theta_{12}>0$ and $\Delta m_{31}^{2}>0$ and the one with $\cos 2 \theta_{12}<0$ and $\Delta m_{31}^{2}<0$. This degeneracy is the result of the symmetry under transformations in (2.2) when matter effects are neglected. The subdominant matter effects slightly lift this degeneracy but not enough to render them distinguishable. In the next section, we discuss whether alternative methods to determine $\operatorname{sign}\left(\Delta m_{31}^{2}\right)$ based on matter effects by long baseline experiments or atmospheric neutrino experiments can lift this degeneracy. The LMA-Dark solution can be tested by neutrino scattering experiments sensitive to NSI effect. Similar discussion can be repeated for figure 2 where the LMA-Dark solution is taken as the true solution.

A similar discussion also applies for inverted hierarchy: contours for inverted hierarchy with $\cos \left(2 \theta_{12}\right)>0$ and $\cos \left(2 \theta_{12}\right)<0$ are very similar respectively to figure 2 and figure 1 .

\section{$5 \quad$ Degeneracy and matter effects}

In this section, we generalize the symmetry under transformation shown in eq. (2.2) to all oscillation modes taking into account the matter effects on oscillation. A similar approach 

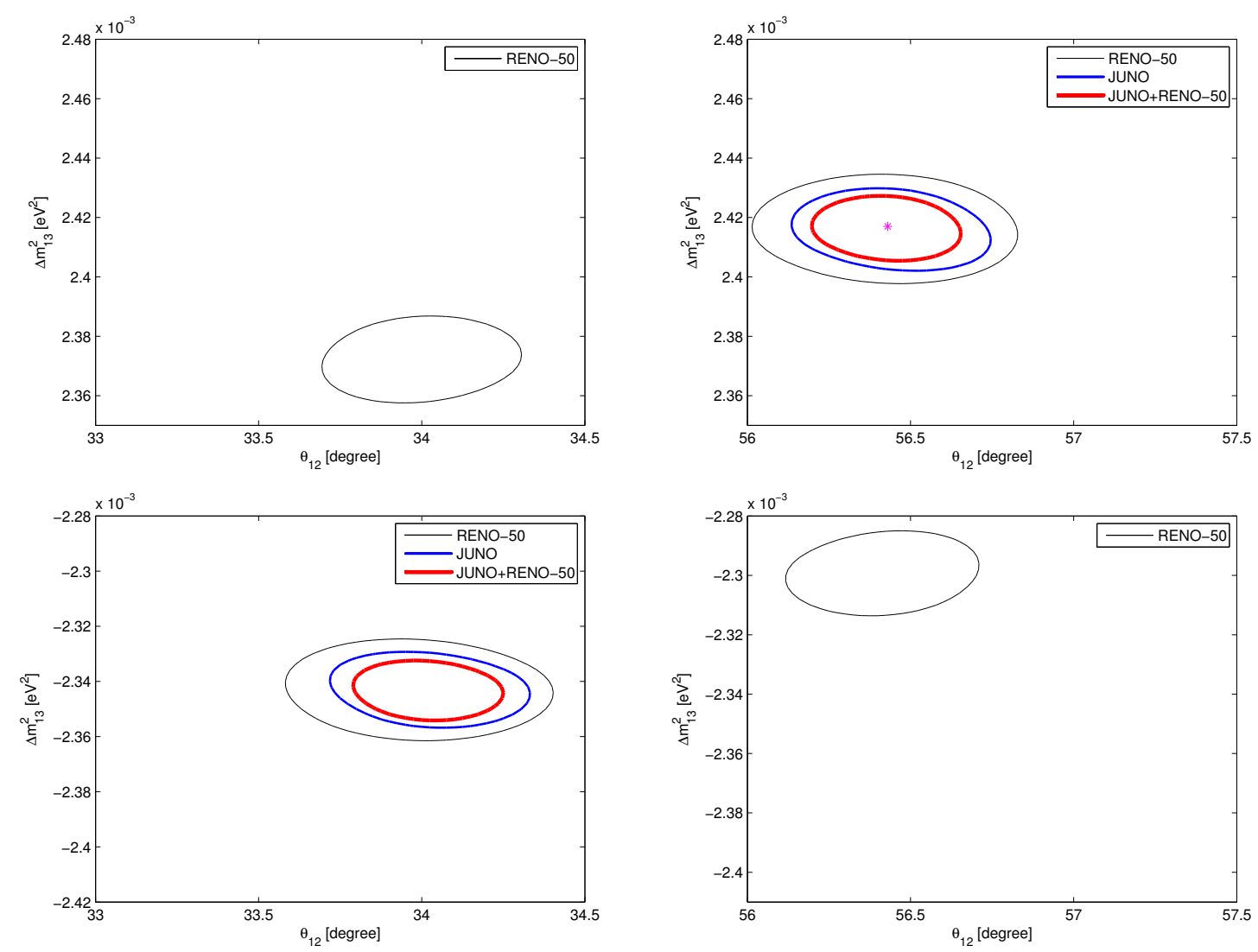

Figure 2. The same as figure 1 except that we have taken the true values to be $\Delta m_{31}^{2}=2.417 \times$ $10^{-3} \mathrm{eV}^{2}$ and $\theta_{12}=56.43^{\circ}$. That is we have taken the LMA-dark solution instead of the standard LMA solution.

is also taken in [6]. The effective Hamiltonian governing the evolution of neutrino states in the presence of matter effects can be written as

$$
H=V_{\text {vacc }}+V_{\text {eff }} \text { where } V_{\text {vacc }}=U_{P M N S} \cdot \operatorname{Diag}\left(\Delta_{1}, \Delta_{2}, \Delta_{3}\right) \cdot U_{P M N S}^{T},
$$

in which $\Delta_{i}=m_{i}^{2} /\left(2 E_{\nu}\right)$ and

$$
U_{P M N S}=\left[\begin{array}{ccc}
c_{12} c_{13} & s_{12} c_{13} & s_{13} e^{i \delta} \\
-s_{12} c_{23}-c_{12} s_{23} s_{13} e^{i \delta} & c_{12} c_{23}-s_{12} s_{23} s_{13} e^{i \delta} & s_{23} c_{13} \\
s_{12} s_{23}-c_{12} c_{23} s_{13} e^{i \delta} & -c_{12} s_{23}-s_{12} c_{23} s_{13} e^{i \delta} & c_{23} c_{13}
\end{array}\right]
$$

$V_{\text {eff }}$ is a matrix describing both standard and non-standard matter effects. For the standard case $V_{\text {eff }}$ is diagonal with $\left(V_{\text {eff }}\right)_{\mu \mu}=\left(V_{\text {eff }}\right)_{\tau \tau}$. Replacing $\theta_{12} \rightarrow \pi / 2-\theta_{12}, \delta \rightarrow \delta+\pi$ and $\Delta_{1} \leftrightarrow \Delta_{2}, V_{\text {vacc }}$ will transform into $S \cdot V_{\text {vacc }} \cdot S$ where $S=\operatorname{Diag}(1,-1,-1)$. Since we have the freedom of rephasing $\nu_{\alpha}$, the oscillation probabilities will remain the same provided that at the same time, $V_{\text {eff }} \rightarrow S \cdot V_{\text {eff }} \cdot S$; i.e., $\left(V_{\text {eff }}\right)_{e \mu} \rightarrow-\left(V_{\text {eff }}\right)_{e \mu}$ and $\left(V_{\text {eff }}\right)_{e \tau} \rightarrow-\left(V_{\text {eff }}\right)_{e \tau}$. Replacing $\Delta_{1} \leftrightarrow \Delta_{2}$ is equivalent to $\Delta_{21} \rightarrow-\Delta_{21}$ and $\Delta_{31} \rightarrow \Delta_{31}-\Delta_{21}$. On the other hand the evolutions with $H$ and $-H^{*}$ lead to the same oscillation probabilities [6]. Thus, 
the oscillation probability will be the same if we simultaneously replace

$$
\theta_{12} \rightarrow \frac{\pi}{2}-\theta_{12}, \quad \delta \rightarrow \pi-\delta, \quad \Delta_{31} \rightarrow-\Delta_{13}+\Delta_{21} \quad \text { and } \quad V_{\mathrm{eff}} \rightarrow-S \cdot V_{\mathrm{eff}} \cdot S
$$

Notice that the transformation in eq. (2.2) is a subset of these transformations. Since for reactor neutrinos, $\delta$ and matter effects $\left(V_{\text {eff }}\right)$ are irrelevant, we did not need to include the transformations of $\delta$ and $V_{\text {eff }}$ in eq. (2.2). Within the SM, $V_{\text {eff }}$ is fixed by the composition of the medium and the Fermi constant: $\left(V_{\text {eff }}\right)_{e e}=\sqrt{2} G_{F} N_{e}-\sqrt{2} G_{F} N_{n} / 2$ and $\left(V_{\text {eff }}\right)_{\mu \mu}=$ $-\sqrt{2} G_{F} N_{n} / 2$. As a result, replacing $V_{\text {eff }} \rightarrow-S \cdot V_{\text {eff }} \cdot S$ is meaningless. However in presence of NSI for a given matter composition, such transformation can be interpreted as shifts in values of $\epsilon_{\alpha \beta}$ which parameterizes new physics. Following [6], let us focus on NSI with $u$ and $d$-quarks parameterized respectively by $\epsilon_{\alpha \beta}^{u}$ and $\epsilon_{\beta \alpha}^{d}$. The effect of NSI on neutrino oscillation in an electrically neutral medium is described [6] by

$$
\epsilon_{\alpha \beta}=Y_{u} \epsilon_{\alpha \beta}^{u}+Y_{d} \epsilon_{\alpha \beta}^{d}
$$

where $Y_{u}=2+Y_{n}$ and $Y_{d}=1+2 Y_{n}$ in which $Y_{n}$ is the neutron to electron ratio. For Long baseline experiments, $Y_{n}=1.012[6,44]$. The fact that the $Y_{n}$ composition of the Sun and Earth are different can help us to partially solve the degeneracy.

Ref. [6] has made a global analysis of data and has found that at $3 \sigma$ C.L., the allowed range of $\epsilon$ for the LMA solution with $\cos 2 \theta_{12}>0$ is

$$
-0.6<\epsilon_{e e}-\epsilon_{\mu \mu}<4
$$

and for the LMA-Dark solution with $\cos 2 \theta_{12}<0$, the allowed range is

$$
-8<\epsilon_{e e}-\epsilon_{\mu \mu}<-4 \text {. }
$$

As expected, while the LMA-dark solution requires $\epsilon \neq 0$, the LMA solution includes $\epsilon=0$. Without loss of generality we can set $\epsilon_{\mu \mu}=0$ because subtracting a matrix proportional to unit matrix (e.g., $\left.\left(V_{\text {eff }}\right)_{\mu \mu} I\right)$ from $H$ will not affect the oscillation probabilities. With this convention, $V_{\text {eff }} \rightarrow-S \cdot V_{\text {eff }} \cdot S$ corresponds to

$$
\epsilon_{e e}+1 \rightarrow-\left(1+\epsilon_{e e}\right)
$$

Symmetry under transformation in eq. (5.3) therefore implies that the part of LMA solution with $2<\epsilon<4$ cannot be distinguished from LMA-Dark solution with $-4<\epsilon<-6$ and opposite hierarchy by oscillation experiments taking place in the earth (i.e., by reactor, atmospheric and long baseline experiments). However, the rest of the range in eqs. (5.4) and (5.5) can be in principle distinguished by long baseline and atmospheric neutrino experiments sensitive to matter effects on oscillation.

We examined the possibility of solving degeneracy by using the NOvA experiment. Sensitivity of NOvA to NSI had also been discussed in $[45,46]$. We used the GLoBES software to carry out the analysis. Details of the simulation of NOvA experiment is based on $[47,48]$. For true values we have taken $\theta_{12}=33.57$ and set all the NSI parameters to zero; $\epsilon=0$. We have assumed normal hierarchical scheme. We have found that after six years of data taking (i.e., 3 years in neutrino mode and 3 years in antineutrino mode), NOvA can rule out the other solution with opposite sign of $\cos 2 \theta_{12}$ and $\Delta_{31}$ with $\chi^{2}=3.9$ which for 2 dof corresponds to about $85 \%$ C.L. 


\section{Conclusions}

We have examined the potential of the intermediate baseline reactor experiments in discriminating between LMA and LMA-Dark solutions. This method is based on determining $\operatorname{sign}\left(\cos 2 \theta_{12}\right)$ rather than probing the NSI. Sensitivity to $\operatorname{sign}\left(\cos 2 \theta_{12}\right)$ (i.e., LMA versus LMA-Dark solutions) as well as to $\operatorname{sign}\left(\Delta m_{31}^{2}\right)$ (i.e., normal versus inverted mass ordering) both appear in oscillatory terms in the survival probability, $P\left(\bar{\nu}_{e} \rightarrow \bar{\nu}_{e}\right)$ that are given by $\Delta m_{31}^{2}$ and are suppressed by $s_{13}^{2}$. Thus, to disentangle their effects, the following challenges have to be overcome: (1) the statistics should be high enough; (2) the energy resolution, $\delta E_{\nu} / E_{\nu}$, should be small enough to resolve the oscillatory terms given by $\left(\Delta m_{31}^{2} L / E_{\nu}\right)$ and (3) the effects of oscillatory terms given by $\Delta m_{31}^{2}$ should not be washed out by averaging over baselines of various reactor cores contributing to the flux. These conditions will be fulfilled at the JUNO and RENO-50 experiments. We have found that for a given hierarchy RENO-50, JUNO and combined RENO-50 and JUNO results can discriminate between LMA and LMA-Dark solution, respectively, at $>90 \%$ C.L., $\sim 3 \sigma$ C.L. and $\sim 4 \sigma$ C.L. after five years.

We have demonstrated that neglecting the matter effects, $P\left(\bar{\nu}_{e} \rightarrow \bar{\nu}_{e}\right)$ becomes symmetric under transformation in eq. (2.2). This means there is a degeneracy between solutions for which both the mass hierarchy and the sign of $\cos 2 \theta_{12}$ are simultaneously flipped. Matter effects can to some extent lift this degeneracy but not enough in order for JUNO and RENO-50 to resolve this degeneracy. Moreover, when we allow a shift in values of NSI parameters, the symmetry can be generalized to include matter effects as described in eq. (5.3). The degeneracy can be partially solved by combining data from long baseline experiments sensitive to matter effects and the solar neutrino data thanks to the fact that the medium in the Sun and in the Earth have different compositions i.e., neutron to electron ratio. In particular, we found that after six years of data taking, the NOvA experiment can discriminate between the LMA solutions with $\cos 2 \theta_{12}>0$ and no NSI $(\epsilon=0)$ and the LMA-Dark solution with opposite mass ordering with about $85 \%$ C.L. Moreover experiments probing neutral current NSI such as neutrino scattering experiments can test LMA-Dark solution and hence break this degeneracy.

\section{Acknowledgments}

The authors are grateful to T. Schwetz, J. Evslin and M. Maltoni for useful remarks. Y.F. acknowledges partial support from the European Union FP7 ITN INVISIBLES (Marie Curie Actions, PITN- GA-2011- 289442).

Open Access. This article is distributed under the terms of the Creative Commons Attribution License (CC-BY 4.0), which permits any use, distribution and reproduction in any medium, provided the original author(s) and source are credited. 


\section{References}

[1] Y. Farzan, S. Pascoli and M.A. Schmidt, AMEND: A model explaining neutrino masses and dark matter testable at the LHC and MEG, JHEP 10 (2010) 111 [arXiv:1005.5323] [INSPIRE].

[2] T. Ohlsson, Status of non-standard neutrino interactions, Rept. Prog. Phys. 76 (2013) 044201 [arXiv: 1209.2710] [INSPIRE].

[3] O.G. Miranda, M.A. Tortola and J.W.F. Valle, Are solar neutrino oscillations robust?, JHEP 10 (2006) 008 [hep-ph/0406280] [INSPIRE].

[4] O.G. Miranda, M.A. Tortola and J.W.F. Valle, Robustness of solar neutrino oscillations in the presence of non-standard physics, AIP Conf. Proc. 917 (2007) 100 [INSPIRE].

[5] F.J. Escrihuela, O.G. Miranda, M.A. Tortola and J.W.F. Valle, Constraining nonstandard neutrino-quark interactions with solar, reactor and accelerator data, Phys. Rev. D 80 (2009) 105009 [Erratum ibid. D 80 (2009) 129908] [arXiv: 0907.2630] [INSPIRE].

[6] M.C. Gonzalez-Garcia and M. Maltoni, Determination of matter potential from global analysis of neutrino oscillation data, JHEP 09 (2013) 152 [arXiv:1307.3092] [INSPIRE].

[7] C. Biggio, M. Blennow and E. Fernandez-Martinez, General bounds on non-standard neutrino interactions, JHEP 08 (2009) 090 [arXiv:0907.0097] [INSPIRE].

[8] S. Davidson, C. Pena-Garay, N. Rius and A. Santamaria, Present and future bounds on nonstandard neutrino interactions, JHEP 03 (2003) 011 [hep-ph/0302093] [INSPIRE].

[9] S.-F. Ge, K. Hagiwara, N. Okamura and Y. Takaesu, Determination of mass hierarchy with medium baseline reactor neutrino experiments, JHEP 05 (2013) 131 [arXiv:1210.8141] [INSPIRE].

[10] E. Ciuffoli, J. Evslin and X. Zhang, Mass Hierarchy Determination Using Neutrinos from Multiple Reactors, JHEP 12 (2012) 004 [arXiv: 1209.2227] [INSPIRE].

[11] Y.-F. Li, J. Cao, Y. Wang and L. Zhan, Unambiguous Determination of the Neutrino Mass Hierarchy Using Reactor Neutrinos, Phys. Rev. D 88 (2013) 013008 [arXiv:1303.6733] [INSPIRE].

[12] A.B. Balantekin, H. Band, R. Betts, J.J. Cherwinka, J.A. Detwiler et al., Neutrino mass hierarchy determination and other physics potential of medium-baseline reactor neutrino oscillation experiments, arXiv:1307.7419 [INSPIRE].

[13] M. Blennow and T. Schwetz, Determination of the neutrino mass ordering by combining PINGU and Daya Bay II, JHEP 09 (2013) 089 [arXiv: 1306.3988] [INSPIRE].

[14] E. Ciuffoli, J. Evslin and X. Zhang, Neutrino mass hierarchy from nuclear reactor experiments, Phys. Rev. D 88 (2013) 033017 [arXiv:1302.0624] [INSPIRE].

[15] E. Ciuffoli, J. Evslin, Z. Wang, C. Yang, X. Zhang et al., Advantages of Multiple Detectors for the Neutrino Mass Hierarchy Determination at Reactor Experiments, Phys. Rev. D 89 (2014) 073006 [arXiv:1308.0591] [INSPIRE].

[16] X. Qian, D.A. Dwyer, R.D. McKeown, P. Vogel, W. Wang et al., Mass Hierarchy Resolution in Reactor Anti-neutrino Experiments: Parameter Degeneracies and Detector Energy Response, Phys. Rev. D 87 (2013) 033005 [arXiv: 1208.1551] [InSPIRE].

[17] E. Ciuffoli, J. Evslin and X. Zhang, The Neutrino Mass Hierarchy at Reactor Experiments now that theta13 is Large, JHEP 03 (2013) 016 [arXiv: 1208.1991] [INSPIRE]. 
[18] A. Bandyopadhyay, S. Choubey, S. Goswami and S.T. Petcov, High precision measurements of theta(solar) in solar and reactor neutrino experiments, Phys. Rev. D 72 (2005) 033013 [hep-ph/0410283] [INSPIRE].

[19] A. Bandyopadhyay, S. Choubey and S. Goswami, Exploring the sensitivity of current and future experiments to theta(solar), Phys. Rev. D 67 (2003) 113011 [hep-ph/0302243] [INSPIRE].

[20] S. Choubey, S.T. Petcov and M. Piai, Precision neutrino oscillation physics with an intermediate baseline reactor neutrino experiment, Phys. Rev. D 68 (2003) 113006 [hep-ph/0306017] [INSPIRE].

[21] S.T. Petcov and M. Piai, The LMA MSW solution of the solar neutrino problem, inverted neutrino mass hierarchy and reactor neutrino experiments, Phys. Lett. B 533 (2002) 94 [hep-ph/0112074] [INSPIRE].

[22] J. Learned, S.T. Dye, S. Pakvasa and R.C. Svoboda, Determination of neutrino mass hierarchy and $\theta_{13}$ with a remote detector of reactor antineutrinos, Phys. Rev. D 78 (2008) 071302 [hep-ex/0612022] [INSPIRE].

[23] L. Zhan, Y. Wang, J. Cao and L. Wen, Experimental Requirements to Determine the Neutrino Mass Hierarchy Using Reactor Neutrinos, Phys. Rev. D 79 (2009) 073007 [arXiv:0901.2976] [INSPIRE].

[24] L. Zhan, Y. Wang, J. Cao and L. Wen, Determination of the Neutrino Mass Hierarchy at an Intermediate Baseline, Phys. Rev. D 78 (2008) 111103 [arXiv:0807.3203] [InSPIRE].

[25] P. Bakhti and Y. Farzan, Constraining Super-light Sterile Neutrino Scenario by JUNO and RENO-50, JHEP 10 (2013) 200 [arXiv:1308.2823] [INSPIRE].

[26] A.N. Khan, D.W. McKay and F. Tahir, Sensitivity of medium-baseline reactor neutrino mass-hierarchy experiments to nonstandard interactions, Phys. Rev. D 88 (2013) 113006 [arXiv: 1305.4350] [INSPIRE].

[27] T. Ohlsson, H. Zhang and S. Zhou, Nonstandard interaction effects on neutrino parameters at medium-baseline reactor antineutrino experiments, Phys. Lett. B 728 (2014) 148 [arXiv:1310.5917] [INSPIRE].

[28] P. Huber, M. Lindner and W. Winter, Simulation of long-baseline neutrino oscillation experiments with GLoBES (General Long Baseline Experiment Simulator), Comput. Phys. Commun. 167 (2005) 195 [hep-ph/0407333] [INSPIRE].

[29] P. Huber, J. Kopp, M. Lindner, M. Rolinec and W. Winter, New features in the simulation of neutrino oscillation experiments with GLoBES 3.0: General Long Baseline Experiment Simulator, Comput. Phys. Commun. 177 (2007) 432 [hep-ph/0701187] [INSPIRE].

[30] http://www.mpi-hd.mpg.de/personalhomes/globes.

[31] http://home.kias.re.kr/MKG/h/reno50.

[32] KamLAND collaboration, A. Gando et al., Reactor On-Off Antineutrino Measurement with KamLAND, Phys. Rev. D 88 (2013) 033001 [arXiv:1303.4667] [INSPIRE].

[33] M. Grassi, J. Evslin, E. Ciuffoli and X. Zhang, Showering Cosmogenic Muons in A Large Liquid Scintillator, arXiv:1401.7796 [INSPIRE].

[34] https://wiki.bnl.gov/dayabay/upload/Li9He8.pdf.

[35] J Evslin, private communication. 
[36] S.-H. Seo, talk at International Workshop on "RENO-50" toward Neutrino Mass Hierarchy, 13 June 2013, Seoul National University, Korea.

[37] T. Mueller, D. Lhuillier, M. Fallot, A. Letourneau, S. Cormon et al., Improved Predictions of Reactor Antineutrino Spectra, Phys. Rev. C 83 (2011) 054615 [arXiv:1101.2663] [INSPIRE].

[38] P. Huber, On the determination of anti-neutrino spectra from nuclear reactors, Phys. Rev. C 84 (2011) 024617 [Erratum ibid. C 85 (2012) 029901] [arXiv: 1106.0687] [INSPIRE].

[39] R.N. Cahn, D.A. Dwyer, S.J. Freedman, W.C. Haxton, R.W. Kadel et al., White Paper: Measuring the Neutrino Mass Hierarchy, arXiv:1307.5487 [INSPIRE].

[40] H. Murayama and A. Pierce, Energy spectra of reactor neutrinos at KamLAND, Phys. Rev. D 65 (2002) 013012 [hep-ph/0012075] [INSPIRE].

[41] KamLAND collaboration, K. Eguchi et al., First results from KamLAND: Evidence for reactor anti-neutrino disappearance, Phys. Rev. Lett. 90 (2003) 021802 [hep-ex/0212021] [INSPIRE].

[42] P. Vogel and J.F. Beacom, Angular distribution of neutron inverse beta decay, $\overline{\nu_{e}}+p \rightarrow e^{+}+n$, Phys. Rev. D 60 (1999) 053003 [hep-ph/9903554] [INSPIRE].

[43] M.C. Gonzalez-Garcia, M. Maltoni, J. Salvado and T. Schwetz, Global fit to three neutrino mixing: critical look at present precision, JHEP 12 (2012) 123 [arXiv:1209.3023] [INSPIRE].

[44] A. Dziewonski and D. Anderson, Preliminary reference earth model, Phys. Earth Planet In. 24 (1981) 297.

[45] A. Friedland and I.M. Shoemaker, Searching for Novel Neutrino Interactions at NOvA and Beyond in Light of Large $\theta_{13}$, arXiv:1207.6642 [INSPIRE].

[46] J. Kopp, M. Lindner, T. Ota and J. Sato, Non-standard neutrino interactions in reactor and superbeam experiments, Phys. Rev. D 77 (2008) 013007 [arXiv: 0708. 0152] [INSPIRE].

[47] NOvA collaboration, D.S. Ayres et al., NOvA: Proposal to build a 30 kiloton off-axis detector to study $\nu_{\mu} \rightarrow \nu_{e}$ oscillations in the NuMI beamline, hep-ex/0503053 [INSPIRE].

[48] Off-Axis-Note-SIM-30 T. Yang and S. Woijcicki, Study of physics sensitivity of $\nu_{\mu}$ disappearance in a totally active version of nova detector, Off-Axis-Note-SIM-30. 